\title{
Textile Flame Retardant Review
}

\author{
Hongkun Zhang \\ Tianjin Polytechnic University \\ Tianjin 300160, China \\ Guoliang Sun \\ Tianjin Bohai Chemical Industry Group Plan \&Design Institute \\ Tianjin 300450, China
}

\begin{abstract}
This article discuss know clearly the dominant species of flame retardant, flame retardant mechanism and flame checking technology combine gave an outline of the testing method of flame retardant effect, lodge set look into highly active, low mammalian toxicity, low fume burst size, multifunctional flame retardant textile, it is our struggling heading.
\end{abstract}

Keywords: Flame retardant, Flame retardant mechanism, Flame retardant finishing testing method

\section{Foreword}

Along with people growth in the living standard and security recognizant, the fire retardancy of textile increasingly came in for folk' attention. How to reduce textile burning susceptibility and release burning toxic gas alight by, reduce people life and property damage by, afterwards bring universal attention has reference to lay store by, wherefore set a high value on, hence relate to flame retardant became particularly interested problem of people. Several aspect did gave an outline of flame retardant.

\section{Flame retardant}

Flame retardant was a sort of assistants to bump flammability or fire resistance of combustible or self-extinguish or smoke abatement up(Cui J,Jiang H L, Wu M Y, et al. 2003.). In recent years, along with fire safe standard increasingly exactitude, the dosage all the while submit ascending trend of the global flame retardant( $\mathrm{Ou} \mathrm{Y} \mathrm{X.} \mathrm{2003.),} \mathrm{for} \mathrm{slate} \mathrm{at}$ henceforth five years within, for global flame retardant demand years equal growth rate approve reach $4 \% \sim 5 \%$.

\subsection{The flame retardant mechanism of the flame retardant}

It is through the medium of some mechanism exert his flame retardant acting to flame retardant, as heat absorbing action, cover action, contain chain reaction, gasses diluting effect.

(1) Absorbing action heat absorbing action

Any catch at short notice station liberated heat is limited alight, at hot conditions, flame retardant occur strong endothermic reaction, absorb burning liberated heat alight, depress combustible ostensible temperature down, effectively restrain flammable gas , prevent flaming overspread .

(2) Cover action

Either flame retardant at high temperature be able to form vitroclastic or stable foam mantle, isolate $\mathrm{O}_{2}$, possess heat insulation, oxygen exclusion, constrain fuel gas outward divergent action from, thereby achieve to flame retardant end.

(3) Restrain chain reaction

Flame retardant act on gas-phase combustion region, catch free radical of combustion reaction, thereby constrain propagation of flame from, enkindle regional flame density fell, ultimately enkindle reaction rate fell till terminate.

(4) Gasses diluting effect

Both flame retardant endothermic decomposition tear loose incombustibility gas as nitrogen, carbon dioxide, sulfur dioxide and ammonia, lead flammable gas strength of fibrous material cracking department by dilute to catch hereinafter, or gotten flame kernel department part regional oxygen insufficiency alight to, prevent burning keep on.

(5) Condensed phase flame retardant

Through the medium of flame retardant action, at condensed phase reaction zone, the thermal cracking reaction 
mechanism of the transfer fiber macro chain, urge occur dehydration, condensation, cyclization, cross linkage reaction, till charring, withal increase charring leavings out, reduce flammable gas make by, lead flame retardant at condensed phase exert flame retardant action to.

\subsection{The sort of the flame retardant}

Here mostly introduce press compound sort of sort, both broke into inorganic flame retardant and organo-flame retardant twain broad heading.

(1) Inorganic flame retardant

The primary function of inorganic flame retardant is endothermic , both it possess high stability, dyes-volatilization, fume virulence low and cost low merit, for the moment the favor of folk is increasing. The trend of development of inorganic flame retardant is supply premium product, on the one hand is to fine, hyperfine grain develop, it is to ameliorate size distribution aspect develop to on the other hand(Sun H Z, Longhai Cong. 2004.).

(2) Organo-flame retardant

(i) Halogen flame retardant

The flame retardant action of halogen organo- flame retardant is through the medium of bring heavier-than-air haloids acid forth to sediment be on the outer layer of blazer, dilute environmental air, isolate fresh air, lead by burnable asphyxia to, at the same time haloids acid well enough catch oxyhydrogen,thereby gotten flame decrease to.

(ii) Organophosphorus flame retardant(HÊrold S,Nab B,Schacker O,etal. 2004.)

Both organophosphorus flame retardant liquidity strong, fume quantity large, be prone to hydrolyze and short of thermostability and more shortcoming too gradualness get conquer. It mostly include halogen phosphorus department and non-halogen phosphorus department . Halogen phosphorus department owing to at the same time contain halogen and phosphorus twain genera element, both atmosphere phase and condensed phase at the same time role playing, flame retardant effect in the extreme ideal, it possess volatility low achromatic without smelt, water fast solution gender grade merit, but short of resistance to heat.

\section{Flame retardant finish}

\subsection{Conventional flame retardant means}

(1) Fire-retardant fiber(Chen B. 1999.)

The manufacturing method of the fire-retardant fiber usually approve reduce to hereinafter three species:

(i) Copolymerization farad:

On the building-up process suffer of fiber forming high polymer, do with maintain phosphorus,halogen,sulfur and more flame retardant elementary compound by way of comonomer introduce macrochain suffer,aganin do with such fire retardancy strong substance add to fiber suffer.

(ii) Cross blend farad: Either is should flame retardant join spinning fusing or spun manufacture fire-retardant fiber .

(iii) Graft modification: Used heat of emission, energetic electron beam or chemical booster resolve fiber (or weft) and vinyl type flame retardant monomer occur graft copolymerization, is obtain hold true whereas everlasting flame retardant modified means.

(2) Flame retardant fabric

(i) Padding baking process

The process flow of the method is: Padding $\rightarrow$ preliminary drying $\rightarrow$ baking $\rightarrow$ after treatment. Its padding liquor is flame retardant solution, commonly be formed of flame retardant, cross-linking agent, catalyze, wetting agent and softener, either formulate become water solution or lattices proceed tidy.

(ii) Steep parch dry farad

Process flow is: Steep $\rightarrow$ dryness $\rightarrow$ after treatment. It is hold fabric lay in flame retardant liquor suffer steep hour to it, take out and drying, at times flame retardant finish approve to dyeing with bath proceed.

(iii) Extrusion coating farad(Giraud S,Bourbigot S,Rochery,et al. 2005.)

It is number flame retardant interfusion resin within proceed process. warranty the different of mechanical equipment into knife coating farad, molding extrusion coating farad and recycle extrusion coating farad.

(iv) Spray method

Put out of service conventional equipment art factitious thick curtain, large-scale rug and more merchandise, the flame retardant finish of the approve last of all in a single operation did hand spraying farad. Toward bulkiness surface have 
got figure, tufting, pile cottony fabric, used padding farad dispose surface fuzz figure came in for traumata to, commonly adopt series spray method.

(v) Organic solvent farad

Used organic matter should flame retardant dissolve, then proceed flame retardant finish, it be able to gotten put hour shorten to rights. In course, both it has to be noted that solvent virulence and combustibility.

3.2 New flame retardant means(Pang Z H. 2004.)( Zhang Y K, Li C D. 2003.)( Zhang P T, et al. 2004.)( Liu L W,Guo F,Chen J F. 2004.)(Alcan. 2000.)

(1) Nanometer flame retardant technology

Some nanophase materials possess posses prevent flaming function, take them as flame retardant enroll to flammable material suffer in, utilize its specific size and structure effect of, the combustion performance of the could transfer these flammable material, the stuff of the built of into possess fire resistance material.

(2) Microcapsule flame retardant technology

Do with flame retardant bruise spread become atom aback about, either used organic matter or inorganic matter proceed parcel, form microcapsule flame retardant; or withal specific area prodigious mineral did bearer, the interstice suffer of the should flame retardant adsorption at the soon some inorganic bearer, blistering become weak gelatin capsule flame retardant.

(3) Superfine melt technology

Owing to flame retardant acting exert is by chemical reaction station dominant,isometrical isometric flame retardant,its particle size more smaller, specific area more larger, flame retardant effect more better. Superfine melt no more will from affinity aspect had in view, considering tone know clearly interfacial interaction up, could still evenly spread mer resin suffer about, the mechanical property of the thereby be able to still effectively ameliorate blend composition.

(4) Surface modification technology

Inorganic flame retardant possess compare strong polarity with hydrophilicity,short of the compatibility with non-polar polymer material, interface hard of wellformed coalesced sum gumming.Both that of for ameliorate his and polymer compartment gumming force and interface affinity, it was most for powerful tool among that adopt coupling agent versus his proceed surface treatment.

(5) Smoke abatement technology

Contain halogen high polymer, halogen department flame retardant and antimony onium compounds are primary fume source. Versus contain halogen high polymer high polymer adopt appending smoke suppressor is solve smoky most measure.

(6) Cross linkage technology

The fire retardancy of cross-linked high polymer is far better than linear high polymer, not only be able to decrease burning flammability fusing drippage,as well as be able to transfer surface texture and/or interface structure of cross blend high polymer, gather instrument intensity.( GB/T 5456-1997)

(7) Macromolecule technology

Macromolecule technology is one of the new techniques of flame retardant under consideration, in recent years its research is activity, and procures round robin fruit.

\section{Flame retardant effect testing method}

\subsection{Burning experimentation}

GB/T5456-1997 provision know clearly textile vertical direction sample flame propagation capable menstruation .GB8746-88 provision know clearly textile vertical direction fabric ease of ignition menstruation . GB8745-88 provision know clearly wove fabric surface combustion behavioral menstruation (GB/T5454-1997).

\subsection{Limiting oxygen index farad}

GB/T5454 -1997 provision know clearly textile combustibility test oxygen index oxygen index farad.( Shen K, Zhang A Y. 1995.)

\subsection{Smokiness testing method}

Both here and abroad have got the species dedication instrument payload test, theory more borrowed light penetrate method. 


\subsection{Flame retardant finish thermal analysis}

It was thermogravimetry (TGA) and differential scan calorimeter (DSC) that flame retardant test suffer staple. At thermo analytical technique suffer well enough avail chromatogram-mass spectra chromatography mass spectrometry of, look into stringy pyrolyzate and more.

\subsection{Taper calorimeter instrument test}

Taper calorimeter instrument adopt oxygen consumption theory measure material catch heat release rate alight, Furthermore, it could measure material catch unit area thermal release velocity alight, sample point burnt time, mass loss velocity, fume density, active combustion hot, noxious gas content is parameter.( Yang D X. 1989.)

\section{Long term potential}

\subsection{Textile flame retardant trend of development}

(1) Strengthen the development research of fire-retardant fiber.

Fire-retardant fiber on part of wear, indoor furnishings, transportation and communication, shield and industrial textiles possess comprehensive application. Proceed from the angle of environmental protection, humanity security and flame retardant efficiency; it was forthcoming development trend that exploitation without halogen, highly active, low fume, harmfulless environment amity form drag burnt fiber.

(2) Strengthen flame retardant textile multifunctional research up

For the moment, either majority fire-retardant fiber or fabric hardly possess flame retardant function, insatiability certain departmental special requirement, as flame retardant water repellency, flame retardant oil-repellent, flame retardant antistatic. Now that inland has got report that flame retardant finish fabric is able to at the same time run up to anti-crease effect for.

5.2 Exploitation new style environmental protects flame retardant Henceforth the develop of flame retardant approximately have got hereinafter some kind of trend:

(1)Exploitation highly active, innocuity, impact material property small flame retardant.

(2) Exploitation flame retardant that possess synergistic effect.

(3) The exploitation of series flame retardant possess differ field of application.

(4) The multiple match of the flame retardant. The multiple match technology of flame retardant embody two-sided signification one is differ flame retardant flame retardant of compartment multiple match problem two is flame retardant with different mer compartment assort problem.

\section{Peroration}

21 century is science and technology science and technology science and technology society high speed high speed ostensive time, persons standard of living escalate meanwhile still lay stress on live quality, folk environmental considerations environmental considerations also increasingly advance. Advance the safety feature of textile, prevent and confine fire, look into highly active, low mammalian toxicity, low fume burst, multifunctional flame retardant textile is our struggling heading.

\section{References}

Alcan Chemicals. (2000). Flame retardants: some new developments[J]. Plastics Additives Compounding, 2000, 2(5), 24-27.

Chen B. (1999). Fiber flame retardant state and headway[J]. Silk technology.1999, (3): 31-35.

Cui J,Jiang $\mathrm{H}$ L, Wu M Y, et al. (2003). The actuality and trend of development of the flame retardant [J]. Shandong light industry institute transaction, 2003, 17(1): 14-17.

GB/T 5456-1997. Textile catch vertical direction sample flame propagation capable menstruation[S].

GB/T5454-1997. Textile catch oxygen index farad[S].

GB8745-88. Wove fabric surface combustion behavioral menstruation.

GB8746-88. The menstruation of the wove fabric combustion performance vertical to sample ease of ignition[S].

Giraud S,Bourbigot S,Rochery,et al. (2005). Flame retarded poly-urea with microencapsulated ammonium phosphate for textile coating[J] .Polymer Degradation and Stability, 2005, 88(1): 106-107.

HÊrold S,Nab B,Schacker O,etal. (2004). A new generation of flame retarded polyamides based on phosphinate[C]. Flame Retardant 2004, London:Interscience Publisher, 2004.

Liu L W,Guo F,Chen J F. (2004). The nanometer alumina cream flame retardant surface modification and the 
application of it used in polypropylene[J]. China plastic, 2004,18(2): 74-77.

$\mathrm{Ou}$ Y X. (2003). Abroad flame retardant extend and the improvidence of development of national flame retardant industry [J]. Fine and Dedication chemicals, 2003, 11(2): 4-7.

Ou Y X. (2003). Subtract disaster AND enviromental protect: Flame retardant extend and hour complete move forward into[J]. Security and health, 2003, (13): 53.

Pang Z H. (2004). Material flame retardant new technique and new species[M] . Beijing: chemical industry book concern, 2004.

Shen K, Zhang A Y. (1995). Taper calorimeter instrument CONE and its application[J].Flame retardant material and technology,1995, 9(2): 9-14,19.

Sun H Z, Longhai Cong. (2004). The application and develop of textile flame retardant finish technology[J]. Qiqiha'er university transaction. 2004, (3): 32-35.

Yang D X. (1989). Flame retardant finish. Printing and dyeing, 1989, 15(6 ): 47-54.

Zhang P T ,et al. (2004). The indicate of the application and process of the pure knit[J]. Shandong wove science and technology, 2004, (6):25-27.

Zhang Y K, Li C D. (2003). Nanometer technology and nanometer plastic[M]. Beijing: China light industry book concern, 2003. 